Article

\title{
Conceptual Design of a Laser Driver for a Plasma Accelerator User Facility
}

\author{
Guido Toci ${ }^{1}$, Zeudi Mazzotta ${ }^{2, \dagger}$, Luca Labate ${ }^{3, \ddagger}$, François Mathieu ${ }^{4}$, Matteo Vannini ${ }^{1}$, \\ Barbara Patrizi ${ }^{1}$ (1) and Leonida A. Gizzi ${ }^{3, *}$ \\ 1 Istituto Nazionale di Ottica, Consiglio Nazionale delle Ricerche (CNR-INO), 50019 Sesto F.no, Italy \\ 2 Ecole Polytechnique, CNRS, 91120 Palaiseau, France \\ 3 Intense Laser Irradiation Laboratory, Istituto Nazionale di Ottica, 56124 Pisa, Italy \\ 4 Laboratoire pour l'Utilisation des Lasers Intenses, CNRS, Ecole Polytechnique, 91128 Palaiseau, France \\ * Correspondence: la.gizzi@ino.cnr.it \\ + Current address: Advanced Research Center for Nanolithography (ARCNL), Science Park 106, \\ 1098 XG Amsterdam, The Netherlands \\ $\ddagger$ Current address: Istituto Nazionale di Fisica Nucleare, Sezione di Pisa, Largo Bruno Pontecorvo, 3, \\ 56127 Pisa, Italy
}

Received: 12 June 2019; Accepted: 5 August 2019; Published: 8 August 2019

\begin{abstract}
The purpose of the European project EuPRAXIA is to realize a novel plasma accelerator user facility. The laser driven approach sets requirements for a very high performance level for the laser system: pulse peak power in the petawatt range, pulse repetition rate of several tens of $\mathrm{Hz}$, very high beam quality and overall stability of the system parameters, along with 24/7 operation availability for experiments. Only a few years ago these performances were considered unrealistic, but recent advances in laser technologies, in particular in the chirped pulse amplification (CPA) of ultrashort pulses and in high energy, high repetition rate pump lasers have changed this scenario. This paper discusses the conceptual design and the overall architecture of a laser system operating as the driver of a plasma acceleration facility for different applications. The laser consists of a multi-stage amplification chain based CPA Ti:Sapphire, using frequency doubled, diode laser pumped $\mathrm{Nd}$ or $\mathrm{Yb}$ solid state lasers as pump sources. Specific aspects related to the cooling strategy of the main amplifiers, the operation of pulse compressors at high average power, and the beam pointing diagnostics are addressed in detail.
\end{abstract}

Keywords: laser driven plasma acceleration; Ti:Sapphire lasers; Chirped Pulse Amplification

\section{Introduction}

Laser-driven plasma accelerators will require a substantial scale-up of their performance in order to address user applications. In particular, to meet user requirements, a higher repetition rate of petawatt peak power is expected in the $100 \mathrm{~Hz}$ range and above, implying several $\mathrm{kW}$ of average laser power. Nowadays, such an improvement is made credible by the rapid advancements of the technology in this field. Nonetheless, the possibility of scaling up the existing technology of high peak power lasers to higher average power remains challenging.

Pulsed high energy solid state lasers were recently realized, reaching uninterrupted operation at pulse energy levels of up to approximately $100 \mathrm{~J}$ and pulse repetition rates (PRR) of up to $10 \mathrm{~Hz}$ e.g., DiPOLE100 (Diode Pumped Optical Laser for Experiments) [1] built by the Rutherford Appleton Laboratory (RAL), and the High-Repetition-Rate Advanced Petawatt Laser System (HAPLS) [2] developed by the Lawrence Livermore National Laboratory (LLNL). Systems reaching the petawatt level with pulse energies in the range of tens of J have been realized [3], such as BELLA (Berkeley Lab 
Laser Accelerator) [4], with an average power output of tens of watts. New systems under realization aim to even higher average power levels, such as HAPLS targeting an output power of $300 \mathrm{~W}$. Nonetheless, to address user-level requirements, the average power of ultrafast lasers needs to increase by one or two orders of magnitude.

The purpose of the European project EuPRAXIA [5] is to provide a conceptual design for a compact, user-oriented, plasma accelerator with superior beam quality to enable free electron laser operation in the $\mathrm{X}$-ray range. User requirements for an operational facility imply a significant increase of the laser driver output parameters with respect to systems currently available or under development: pulse peak power in the petawatt range, pulse repetition rate of several tens of $\mathrm{Hz}$, as well as very high beam quality and overall stability of the system parameters.

A description of the architecture of the laser driver was already published in previous work [6], describing the main parameters and technical solutions. This paper complements the previous one by recalling the general lines of the system architecture and addressing some specific points that are particularly critical for the system operation at high average power levels. These aspects are related to the amplifier's geometrical layout, the cooling solution to address the operation at high average power levels, and the laser beam transport-related issues.

\section{Preliminary Laser Design}

The development of the EuPRAXIA laser driver relies on Chirped Pulse Amplification (CPA) in Ti:Sapphire (Ti:Sa). The achievement of the required performance and reliability levels poses stark challenges related to several key elements in the system, and requires the most advanced components under development at the industrial level, along with specially devised solutions.

The implementation of the required plasma acceleration schemes involves the simultaneous use of up to three different laser systems, and the time of arrival of their output pulses on the plasma target must be carefully synchronized down to below $10 \mathrm{fs}$. Moreover, auxiliary laser beams for diagnostics and a photocatode laser are included, tightly synchronized with the main laser pulses.

The overall architecture of the EuPRAXIA laser system is shown in Figure 1. It consists of three different laser chains, with different output pulse parameters matched to meet requirements for the laser-driven plasma injector at $150 \mathrm{MeV}$ (Laser 1), the laser-driven plasma injector at $1 \mathrm{GeV}$ (Laser 2), and the accelerator stage at $5 \mathrm{GeV}$ (Laser 3). They share a common master oscillator for synchronization purposes. Each one consists of a front end segment, an amplification section, a beam shaping segment, a compressor, and the final transport system to the target.

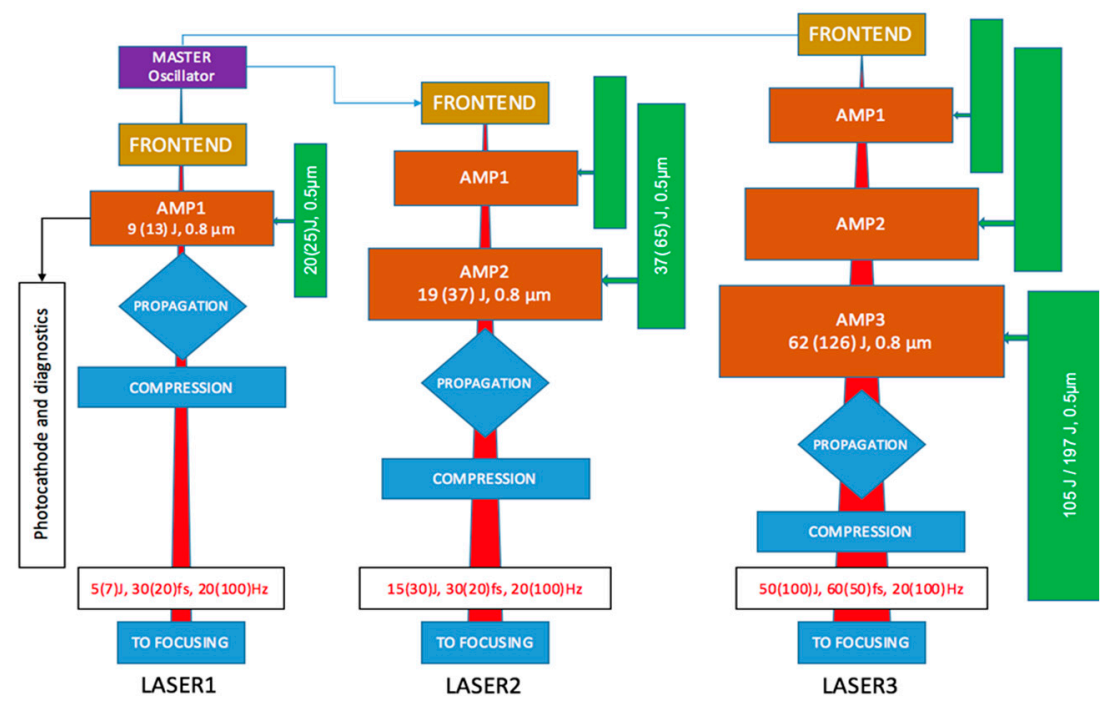

Figure 1. Block diagram of the three laser chains. 
In our study, the most critical points in the of design laser systems turned out to be the following:

(1) the pump lasers for the Ti:Sa amplifiers, in particular regarding the pulse repetition rate (PRR);

(2) the thermal load management, and the optimization of the extraction efficiency for the Ti:Sa amplifiers;

(3) the design of the pulse compression system, mainly in relation to the optical damage and the thermal management of the gratings.

For each laser system, two levels of performance have been considered, with different energy output and PRR (see Table 1), as follows:

(a) Minimum performance level (named P0), featuring lower pulse energy and $20 \mathrm{~Hz} \mathrm{PRR}$;

(b) High performance level (called P1), with higher energy at a PRR of $100 \mathrm{~Hz}$.

Our design shows that the less challenging P0 performance level can be implemented with currently available technologies, requiring mainly integration efforts. Conversely, level P1 lies one step ahead with respect to the current technological capabilities, in particular regarding the PRR of the pump sources. Indeed, the choice of $100 \mathrm{~Hz}$ PRR was found to be the trade-off between the need for a sufficiently high repetition rate for user operation of the facility and the high technology readiness level (TRL) of the required components, assuming a 5-year time to construction.

Table 1. Laser emission parameters for the three devices envisaged for laser plasma injection and acceleration, at the levels of performance of $\mathrm{P} 0$ and $\mathrm{P} 1$. The maximum output energy (before transport and compression) has been calculated from the requirements of energy on target, assuming an overall efficiency of $80 \%$ of the compression and the beam transport system. For Laser 1, a further amount of energy output has been considered for the diagnostic beams (2.5 J at P0 and $3.8 \mathrm{~J}$ at P1).

\begin{tabular}{|c|c|c|c|c|c|c|c|}
\hline \multirow[b]{2}{*}{ Parameter } & \multirow[b]{2}{*}{ Label } & \multicolumn{2}{|c|}{$\begin{array}{l}\text { Laser 1-Injector } \\
150 \mathrm{MeV}\end{array}$} & \multicolumn{2}{|c|}{$\begin{array}{l}\text { Laser 2-Injector } \\
1 \mathrm{GeV}\end{array}$} & \multicolumn{2}{|c|}{$\begin{array}{c}\text { Laser } 3 \\
\text { LPA-Driver } \\
5 \mathrm{GeV}\end{array}$} \\
\hline & & P0 & P1 & P0 & $\mathbf{P 1}$ & P0 & P1 \\
\hline Wavelength (nm) & $\lambda$ & 800 & 800 & 800 & 800 & 800 & 800 \\
\hline Maximum energy on target (J) & Et $t_{\text {arget }}$ & 5 & 7 & 15 & 30 & 50 & 100 \\
\hline Maximum output energy (J) & $E_{\text {out }}$ & 8.8 & 12 & 18.8 & 40 & 62.5 & 130 \\
\hline $\begin{array}{l}\text { Energy tuning resolution } \\
\left(\% \text { of } \mathrm{E}_{\mathrm{out}}\right)\end{array}$ & $\mathrm{dE}$ & 7 & 5 & 7 & 5 & 7 & 5 \\
\hline $\begin{array}{l}\text { Shortest pulse length }\left(\mathrm{FWHM}^{*}\right) \\
(\mathrm{fs})\end{array}$ & $\tau$ & 30 & 20 & 30 & 20 & 60 & 60 \\
\hline Repetition rate $(\mathrm{Hz})$ & $\mathrm{f}$ & 20 & 100 & 20 & 100 & 20 & 100 \\
\hline Energy stability (RMS **)\% & $\sigma_{<\mathrm{E}>}$ & 1 & 0.6 & 1 & 0.6 & 1 & 0.6 \\
\hline
\end{tabular}

* FWHM: Full Width Half Maximum, ${ }^{* *}$ RMS: Root Mean Square.

\subsection{Front End}

The design of the front-end segment, either at the performance level of $\mathrm{P} 0$ or P1, is not critical. Several devices that are also commercially available can be adopted. The primary source is a common master oscillator, whose output is shared among the three systems. Then, a first CPA stage amplifies the pulse at the $\sim 1 \mathrm{~mJ}$ level, in order to efficiently pump an XPW stage immediately downstream. The resulting pulse, with typical energy in the order of 10-100 $\mu \mathrm{J}$, is then seeded into the main CPA chain. A regenerative amplifier and two multi-pass amplifiers increase the energy up to approximately $>\sim 1 \mathrm{~J}$, which is needed to seed the first stages of the three amplification chains.

During the amplification, the pulse spectrum will be slightly narrowed (few percent of reduction in the FWHM of the spectrum) and red shifted (few $\mathrm{nm}$ ), due to the higher gain experienced by the leading front of the pulse (which corresponds to the red end of the pulse spectrum due to the chirping). These effects were calculated in the simulations and they will be pre-compensated at the level of the seed pulse by blue-shifting and broadening its spectrum to obtain the desired peak wavelength and 
pulse duration at the output of each amplification chain. For this purpose, each chain has its own front end stage. The master oscillator will generate pulses with shorter duration and broader bandwidth than that actually required for the pulse amplification; the pulse sent to each front-end stage will be spectrally filtered to obtain the peak wavelength and bandwidth required by each amplification chain.

\section{Options for Pulse Train Generation}

One of the acceleration schemes proposed in order to obtain the high quality electron bunches required for EuPRAXIA is the so-called Resonant Multi-Pulse Ionization injection (ReMPI) scheme [7]. In this scheme, the wakefield is resonantly excited by a train of (relatively low intensity) pulses at the Ti:Sapphire (TiSa) fundamental wavelength, while ionization injection is driven by a shorter wavelength (second or third harmonic) pulse. To this purpose, a small fraction of the laser pulse is picked up and frequency doubled or tripled by a nonlinear crystal, while the most energetic fraction (almost 97\% of the total energy, in the case being studied for EuPRAXIA) is shaped temporally as a sequence of sub-pulses. Over the past few years, a few optical schemes were proposed in order to produce a train of ultrashort pulses from a CPA TiSa chain [8-10]. However, in their proposed variants these schemes do not meet the two essential requisites for driving the ReMPI acceleration scheme within EuPRAXIA, namely a high efficiency (energy throughput into the pulse train) and reduced amplitude variations among the different pulses of the train. Within the EuPRAXIA laser design, we are thus exploring other optical schemes able to meet these requirements. Here we sketch the two approaches we are pursuing, briefly outlining their pros and cons.

A first scheme exploits a segmented delay mask, made up of concentric rings of thin fused silica plates with different thicknesses. Full simulations accounting for both the temporal and spatial (that is, focusing) issues were carried out, which were reported in previous work [11]. Of course, the beam propagation optics upstream of the last focusing parabola must be designed taking into account the presence of the delay mask. While very easy to setup, the main drawback of this optical scheme is the small differences among the focal spot sizes and the Rayleigh ranges expected for the different pulses. However, theoretical estimates show that these variations can be tolerated in the ReMPI scheme.

The study of a second approach was undertaken more recently, relying on a slight variant of an optical scheme proposed in a previous study [9]. The concept (named "quasi lossless Train generation by an Early aMplitude dIvision" (TEMPI)) is based upon the usage of birefringent plates of increasing (doubling) thicknesses and crossed polarization. In our scheme the stack of birefringent plates is used early (i.e., upstream) in the laser amplification chain (on the stretched pulse), so that (a) small aperture optics can be used for this purpose and (b) the energy loss (50\%) due to the polarization rejection can be recovered at a much smaller price in terms of pump energy (see later). Optical simulations of the amplification process with this scheme have been carried using the MIRO (Mathématiques et Informatique pour la Résolution des problems d'Optique-Mathematics and computer science to solve optical problems) code [12] in order to assess the importance of the interference and nonlinear effects occurring along the chain for the EuPRAXIA Laser 1 (see Figure 1), which could possibly be used as the injector with the ReMPI scheme. The simulation is based on a slowly varying envelope approximation computational scheme-the pulse is modeled as an amplitude profile (with dependence on both time and spatial coordinates) and a time varying phase profile modulating a carrier frequency, with the assumption that the variation of the amplitude and phase envelopes are very small on the spatial scale of the wavelength and of the time scale of the optical period. In particular, simulations were carried out for a ReMPI acceleration regime and using four pulses, with duration of $30 \mathrm{fs}$ and separation of the pulses over approximately $103 \mathrm{fs}$. A detailed discussion of the results of these simulations is beyond the scope of this paper and will be provided elsewhere. Here, we only mention that according to these simulations, the accumulated B-integral for Laser 1 is $\sim 0.4$, while the maximum intensity in the chain is $\sim 1.1 \times 10^{9} \mathrm{~W} / \mathrm{cm}^{2}$, which can be considered as quite safe values; this is due to the original dimensions of the laser amplifier (see later), which, among other issues, were aimed at minimizing nonlinear effects. On the other hand, as it can be easily realized, Kerr nonlinearity in the crystal and 
gain saturation and narrowing result (due to the splitting and delaying of the original stretched pulse) in the appearance of pre- and post-pulses between the main pulses of the pulse train (with respect to the desired number of pulses), and to some imbalance in the peak power levels of the amplified pulses. In the case studied for an EuPRAXIA injector, using a ReMPI scheme with a 4-pulse train produced by Laser 1, the maximum intensity of these spurious pulses was $<\sim 0.2$ of the intensity of the main pulses in the train; the overall energy content in the spurious pulses was about $13 \%$ of the overall pulse train, with $2 \%$ of the total energy located in pre-pulses before the train. The fluctuation between the peak power levels of the main pulses after the amplification was about $\pm 3 \%$. Particle in cell (PIC) simulations were recently performed using the actual pulse train temporal profile resulting from the simulations on the amplification (which will be presented elsewhere), and they show no major effects on the plasma wave excitation and final bunch quality from these smaller intensity pulses and the small variation in the peak power of the individual pulses. Nonetheless, these spurious effects can be possibly mitigated by proper temporal, spectral, and phase shaping of the seed pulse train before of the amplification. This will be the subject of future studies. In the case considered here, the cost in terms of additional pump energy (needed to recover the 50\% energy loss in the plate/polarizer array, so as to keep the extraction efficiency unchanged) has been estimated to be about 4J, over a total pumping of about $35 \mathrm{~J}$ for the front end and the first stage (see below).

\subsection{Power Amplification}

Pulse amplification relies on CPA in Ti:Sa, requiring pump sources in the visible range (i.e., frequency doubled, solid state $\mathrm{Nd}$, or $\mathrm{Yb}$-based lasers with emission in the range $515-532 \mathrm{~nm}$ ). Several devices have been realized, achieving amplification of ultrashort $(<20 \mathrm{fs})$ laser pulses with energy output of up to $100 \mathrm{~J}$ or greater, but these devices operate at a very low PRR $(<1 \mathrm{~Hz}$, or single shot). EuPRAXIA requires a much higher PRR, resulting in high average pump power requirements and a severe thermal load on the amplifiers.

Thermal load issues were addressed by means of water cooling at near room temperature of the end surfaces of the amplification crystals, shaped as disks with a relatively large diameter to thickness ratio, as recently proposed [13]. Alternative approaches were considered, such as the cooling of the crystals by means of a high speed gas flow at cryogenic temperatures, as implemented in the DIPOLE Yb:YAG high energy laser system [14,15], or more recently in the Ti:Sapphire high energy amplifier implemented in the ELI-HAPLS system [16]. This cooling method was nonetheless considered not suitable for this design, as it cannot provide a sufficient heat removal for this application, and it can hardly be scaled up to even higher thermal loads, as will be clarified in the following parts. For this reason, this solution was not further studied in the conceptual design.

The optimization of the extraction efficiency was carefully considered to reduce both the requirements on the pump lasers and the overall thermal load. This was addressed by a careful repartition of the amplification between the various stages, and by implementing the Extraction During Pumping (EDP) method to limit the buildup of transverse gain, and thus transverse parasitic lasing $[17,18]$.

Modularity and scalability were addressed in the design — the various laser chains are built by different combinations of a limited set of amplification modules: Laser 1 (see Figure 1) is composed of a single amplification stage (AMP1); Laser 2 consists of two amplification stages (AMP1, featuring the same design and operating parameters as in Laser 1, and AMP2); finally, Laser 3 consists of three stages (AMP1, AMP2, and AMP3), the first two being identical to Laser 2, and the third one featuring a dedicated design. The modules are already dimensioned to operate at the P1 level (Table 1) if a sufficient pump pulse energy is available, as will be clarified in the following sections. This approach is advantageous in view of an industrial development of the system and in view of the scaling up of its performance from P0 to P1 along its lifetime. 


\subsection{Ti:Sapphire Amplifiers Structure and Geometry}

A dedicated analysis was carried out to define the geometrical layout of the multi-pass amplifier based on thin disk crystals. To achieve sufficient pump absorption and energy storage, several disks (from 2 to 4) must be used in each amplification stage, depending on the pump energy and PRR. To achieve efficient amplification and energy extraction the amplified beam must cross the crystal several times (from 4 to 6 depending on the configuration).

As for the cooling strategy and amplification scheme, two possible approaches were considered for the disks: a transmission geometry and a reflection geometry.

In the transmission geometry, the disk-shaped crystal is cooled on both faces; both the pump beams and the amplified beam cross the crystal, the cooling water flow, and the flow containment windows. This solution (proposed in a previous study [13] and analyzed in another study [6] for the Eupraxia system) offers a good performance in terms of heat extraction and allows implementation of a simpler layout from a geometrical point of view, but it presents a potential drawback because the amplified beam is potentially subjected to optical perturbations due to the turbulence of the cooling fluid.

In the reflection geometry, one of the faces of the crystal is highly reflective for the amplification and pump beam. The amplified beam and the pump beams enter the crystal from the front face and are reflected back in the incoming direction on the cooled back surface (Figure 2). As the laser beams do not cross the cooling flow, no optical perturbations are possible. This arrangement allows for less favorable surface/volume ratio for cooling and requires a more complex geometry.

Here, we describe in more detail the design of the amplifiers for the reflection geometry. Both design approaches are still under consideration, as it is difficult to assess the balance of the respective pros and cons only on the basis of theoretical considerations and simulations, and it will be addressed by suitable pilot studies.

The various laser modules were dimensioned by means of numerical simulations using the code MIRO developed by CEA (Commissariat à l'énergie atomique et aux énergies alternatives, France) [12], validated by comparison with similar existing real laser systems [18,19]. As a baseline design, all of the amplifying modules feature a multi-pass amplification architecture with 4 passes. Here, we provide details of the amplifier AMP3 (see Figure 1), as this is the amplifying unit running at the highest energy and power level, and thus is the most challenging in terms of thermal management. The unit consists of 3 equal amplification disks. The main parameters are reported in Table 2 for the performance levels $\mathrm{P} 0$ and P1.

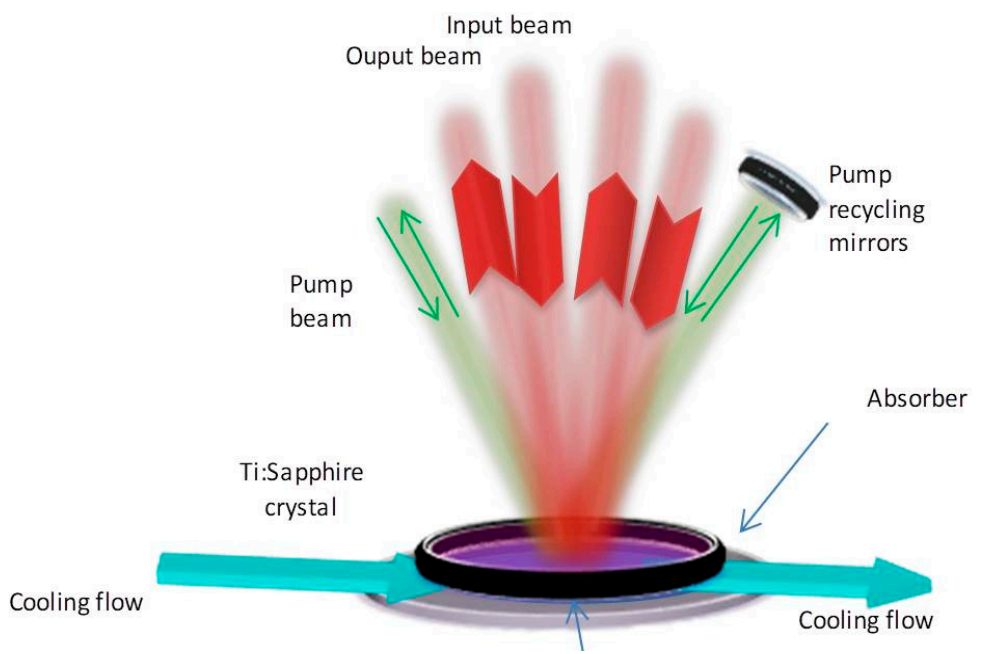

Figure 2. Layout of a single amplifying element in the reflection geometry. 
Table 2. Main design and operational parameter for the amplifier AMP3 in the reflection configuration.

\begin{tabular}{llll}
\hline \multicolumn{1}{c}{ Parameter } & Value & Parameter & Value (P0-P1) \\
\hline Crystal diameter $(\mathrm{cm})$ & 16 & Beam diameter FWHM $(\mathrm{cm})$ & $12-13$ \\
Crystal clear aperture $(\mathrm{cm})$ & 13.2 & Pump beam energy $(\mathrm{J})$ & $109-189$ \\
Crystal thickness $(\mathrm{mm})$ & 10.7 & Seed pulse energy $(\mathrm{J})$ & $18.8-40$ \\
Crystal doping $(\% \mathrm{Wt})$ & 0.045 & Output pulse energy $(\mathrm{J})$ & $62.0-130$ \\
& & Pulse bandwidth $(\mathrm{nm} . \mathrm{FWHM})$ & $27-23$ \\
\hline
\end{tabular}

The geometrical layout of the disk arrangement is exemplified in Figure 3, which shows separately the amplified beam path and the pump beam path. The seed pulse enters the first disk of the chain, and it is routed to the second and third disk by a suitable steering mirrors arrangement. After a first pass along the disk sequence, the pulse travels along a delay line and then it is sent backward along the chain meeting the three disks in reverse order. Angular multiplexing is used to separate the forward and the backward beam paths.

The three disks are pumped with the same amount of energy, equally distributed from the same pump pulse by beam-splitters (Figure 3 right). On each disk, the pump energy is completely absorbed in four passes. The path of the pump beams is slanted in the vertical (z) plane to avoid obstruction by the steering mirrors for the amplified beam. The distance between the steering mirrors and the Ti:Sa disks is about $4 \mathrm{~m}$, and the angles of the amplified beam path with respect to the disks' surfaces are $1.2^{\circ}$ and $2.4^{\circ}$.
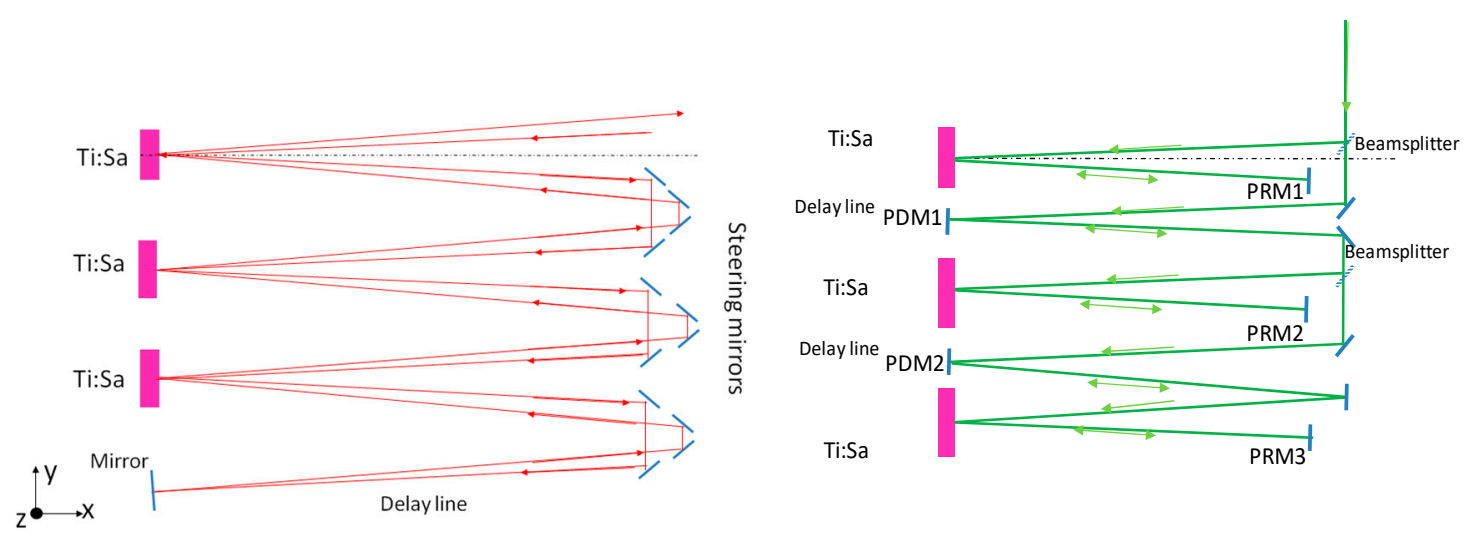

Figure 3. (Left) amplified beam path through the Ti:Sapphire disks. Pump beams are not represented for clarity. (Right) pump beam path. Drawings not in scale, with angles exaggerated for clarity.

The pump beam path includes suitable delay lines so that the arrival of the pump pulse on each disk is synchronized with the arrival of the amplified pulse.

The energy amplification process in AMP3 is described in Figure 4, which reports the pulse energy for increasing numbers of passes, for the performance levels P0 and P1. For the P0 level the module is seeded with $18.8 \mathrm{~J}$ and pumped with $109 \mathrm{~J}$, resulting in a output energy of $62 \mathrm{~J}$; for the P1 performance level the output energy is $130 \mathrm{~J}$ with a seed pulse energy of $40 \mathrm{~J}$, and a pump energy of $189 \mathrm{~J}$.

The amplification process takes advantage of the fact that in both cases the seed energy is relatively high, so an efficient energy extraction can be realized even though the amplification per bounce on a single disk is relatively low. The different pulse duration and spectral bandwidth between P0 and P1 were found to have a negligible impact on the energy extraction. 


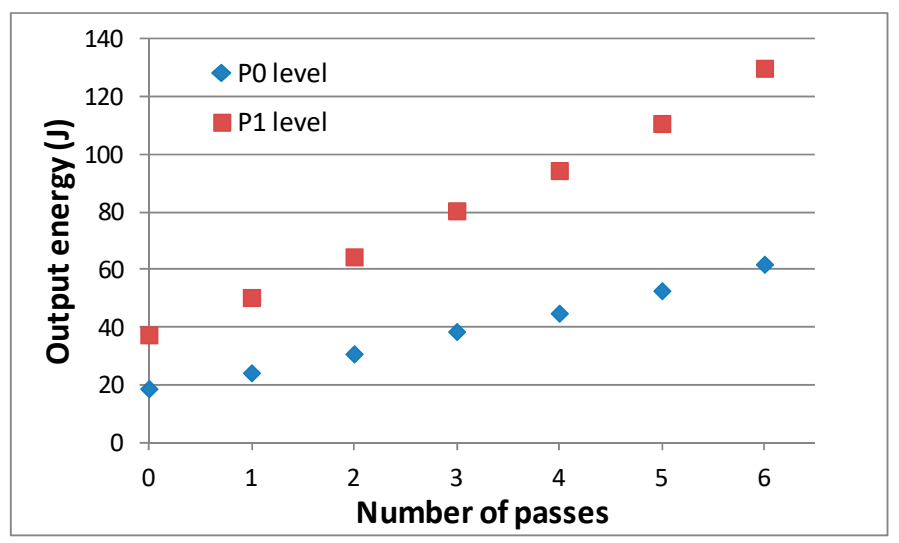

Figure 4. Calculated pulse energy evolution during the propagation in AMP3 at the level of performance of $\mathrm{P} 0$ and P1. Each pass corresponds to a single transit of the pulse in the disk (a single reflection consists of 2 passes).

The same concept was applied in the design of all the three modules (AMP1, AMP2, AMP3), as they operate at similar levels of pump pulse fluence, seed pulse fluence, and stored energy density. Therefore, the amplification of the pulse fluence is similar for all the three modules and it scales in the same way when going from P0 to P1 level. Energy scaling from AMP1 to AMP3 was mainly obtained by increasing the beam diameter at about constant fluence. In some cases a small adjustment in the beam diameter was needed to optimize the process either at P0 or at P1.

Regarding the thermal load, the various modules were designed to safely operate at the performance level P1; at the performance level P0 the thermal load is well below the design limits for each stage.

In conclusion, as was mentioned earlier, the output level of the amplifiers can be scaled up from P0 to P1 by increasing the pump and the seed energy without needing major modifications.

The occurrence of parasitic lasing was carefully analyzed in the design. Details about the methodology followed for this subject can be found in previous work [6]. The choice of crystal diameter, beam size, crystal doping, and thickness result from an accurate tradeoff between longitudinal gain, energy storage, cooling considerations, and transverse lasing control and suppression. We notice here that although the multi-slab approach was primarily adopted to provide enough heat exchange surface area, as a side advantage it allows distribution of the stored energy in several crystals, reducing the transverse gain at the pump input face. Increasing the doping level would be favorable from the thermal point of view (as it would allow reduction of the crystal thickness for the same pump absorption), but it would result in an increased parasitic gain.

The implementation of the extraction during pumping (EDP) strategy relies on the proper synchronization between the injection of the seed pulse and of the pump pulse, and on the presence of the delay lines for the pump beam between the various disks.

On each disk, the pump pulse is almost completely absorbed in four passes, using the pump recycling mirrors PRM1, PRM2, and PRM3 to send back in the crystal the fraction transmitted after the first bounce.

The time required to complete the pump absorption process on each disk from the moment when the leading edge of the pump pulse first hits the disk depends on the pump pulse duration and on the distance between the disk and the PRM. As a numeric example, if the pump pulses are roughly rectangular with a duration of $15 \mathrm{~ns}$, and the distance between the disks and the PRM is $2.5 \mathrm{mt}$, the pump absorption will be completed in about $30 \mathrm{~ns}$ on each disk.

The seed pulse arrives on the disk before the end of the absorption period, so that the available energy for amplification is only the fraction of the pump pulse absorbed before the arrival of the seed pulse itself; after the first bounce, the seed pulse leaves the disk and it returns back only for the return pass. During this interval the energy absorption is completed and the stored energy remains available 
for the amplification in the return pass, as the lifetime of the Ti:Sa is obviously much longer than the overall pulse transit time in the disks sequence.

This sequence of events can be replicated in each disk along the chain by keeping the arrival time of the pump pulse on each disk properly synchronized with the arrival of the seed pulse. For this purpose, the additional pump delay lines between each disk (mirrors PDM1 and PDM2) defer the arrival of the pump pulse on each disk exactly by the time that the seed pulse takes to complete its path through the previous Ti:Sa disks.

This timing scheme is exemplified in the diagram in Figure 5. Through this approach, the stored energy repartition between the first and the second bounce on each disk can be finely tuned by adjusting the injection delay between the pump pulse and the seed pulse, and the length of the intermediate delay lines. As the pump absorption process on each disk takes a time in the order of several tens of ns, a timing accuracy in the range of $\sim 0.3-0.5 \mathrm{~ns}$ can allow a control of the energy repartition with about $1 \%$ accuracy.

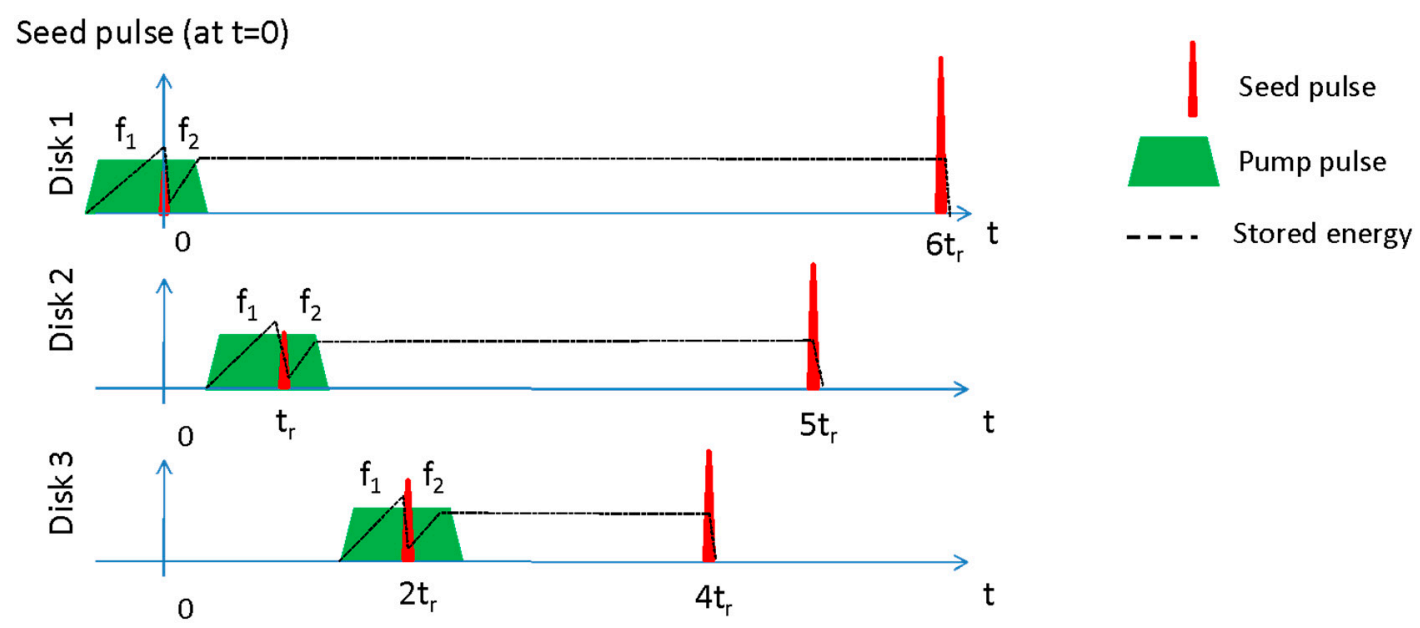

Figure 5. Overview of the synchronization scheme for the implementation of the EDP (Extraction During Pumping) strategy. Red peaks represent the amplified pulses, the green trapezoids represent the arrival of the pump pulses (including recycling), and the dashed line is the stored energy. The time $t_{r}$ is the transit time from one disk to the next one (about $24 \mathrm{~ns}$ for AMP3). The fraction of stored energy $\mathrm{f}_{1}$ available for the amplification in the first transit can be adjusted by finely tuning the delay between the arrival of the amplified pulse and the pump pulse on each disk, leaving the remaining fraction $\mathrm{f}_{2}$ available for the amplification of the second transit.

\subsection{Fluid Cooling Simulations}

A simulation of the temperature distribution and of the thermomechanical stresses affecting the amplifier disks was carried out by means of Finite Element Analysis (FEA) software available in a commercial software package, i.e., LAS-CAD (ver. 3.6.1) developed by LAS-CAD GmbH (www.las-cad.com), to calculate the spatial temperature distribution in the gain material, the resulting stress and deformation distribution induced by the thermal expansion, and finally the thermal aberrations computing the optical path difference (OPD) distribution across the crystal aperture due to both the variation of the refractive index with temperature and the variation in the crystal thickness due to thermal expansion and thermally induced stresses. Thermally induced birefringence [20] was not considered. Detailed results of this approach are described in previous work [6].

A key point to obtain meaningful results from the FEA simulations is the modelling of the heat exchange between the solid and the fluid. This was modelled by means of a film coefficient $k=Q / \Delta T$, where $\Delta T$ is the local difference of temperature between the solid surface and the fluid, and $Q$ is the power per unit surface transferred from the solid to the fluid. Although approximate, this approach 
is very effective in reducing the computational effort to acceptable levels, in particular with full 3D models.

The fluid-solid heat transfer process was studied by means of dedicated fluidodinamical simulations, in 2D geometry, in order to obtain reliable values for the heat transfer coefficient used in the full 3D simulation. The computational method was a numerical solution of the Navier-Stokes equations for mass and energy transport, using the so-called Low Reynolds k- $\varepsilon$ method, which is capable of a quite accurate simulation of the behavior of the fluid-solid interface layer with a reasonable computational effort. The fluidodynamics simulations were carried out using a commercial software package (COMSOL Multiphysics Version 5.3a).

An example is shown in Figure 6, depicting the fluid flow in contact with the back (reflective) surface of the disk of Figure 2. The simulation considers a flow of water at $6 \mathrm{~m} / \mathrm{s}$ entrance speed, in a cooling channel with a thickness of $5 \mathrm{~mm}$. The fluid flow is heated from its bottom side by a constant heat flux of $25 \mathrm{~W} / \mathrm{cm}^{2}$, corresponding to the heat input generated in the Ti:Sapphire disk under pumping at the P1 performance level. The length of the heated portion of the channel is $160 \mathrm{~mm}$ (corresponding to the diameter of the disk of AMP3). Figure 5 shows the calculated fluid velocity distribution. The temperature dependence of the water properties (viscosity, density) was taken into account; buoyancy effects were considered negligible.
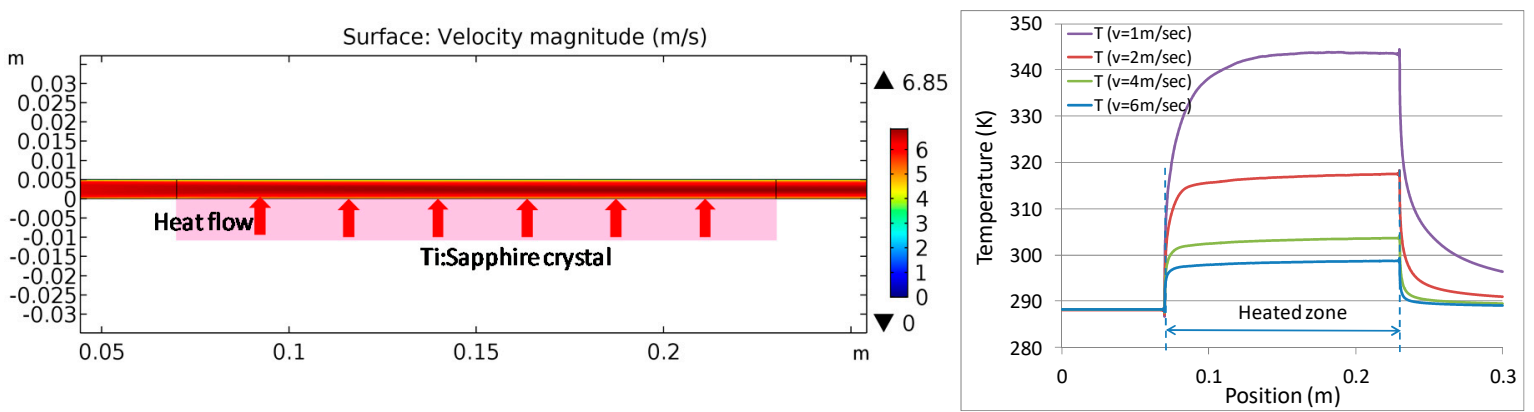

Figure 6. (Left) fluid velocity distribution (in $\mathrm{m} / \mathrm{sec}$ ) in the cooling channel in the simulation conditions specified in the text. The position of the Ti:Sapphire crystal is shown for clarity, but it is considered in the thermal simulation only as a thermal boundary condition for the fluid flow (constant surface heat flux). (Right) Temperature profile along the Ti:Sapphire crystal surface for different flow speeds, at a heat input of $25 \mathrm{~W} / \mathrm{cm}^{2}$, with an input temperature of $288.15 \mathrm{~K}\left(15^{\circ} \mathrm{C}\right)$.

Figure 6 also shows the calculated temperature distribution of the boundary layer of the water flow in contact with the Ti:Sapphire surface for different flow speeds. Under fully developed heat transfer conditions the temperature increase of the surface is about proportional to the heat input. This allows calculation of the effective film coefficient $k=Q / \Delta T$, which expresses the cooling capability of the fluid flow. The effective film coefficient resulted independently from of the heat input, and proportional to the flow speed (proportionality constant of about $0.04 \mathrm{~J} / \mathrm{cm}^{3}$ ). At the maximum flow speed of $6 \mathrm{~m} / \mathrm{sec}$ the film coefficient was $2.45 \mathrm{~W} /\left(\mathrm{cm}^{2} \mathrm{~K}\right)$.

With the channel configuration shown in Figure 6 (i.e., parallel walls), only a very thin layer of fluid near the surface is actually involved in the heat exchange process. Other channel configurations were also studied, with ridges meant to enhance the fluid turbulence and increase the heat exchange, as shown in Figure 7. Due to turbulence, the fluid speed locally increases (up to about twice the input velocity), thus improving the heat transfer from the lower surface. The temperature increase of the heated surface is thus lower by more than a factor of 2 with respect to the previous case, with a corresponding increase in the film coefficient $k$. 

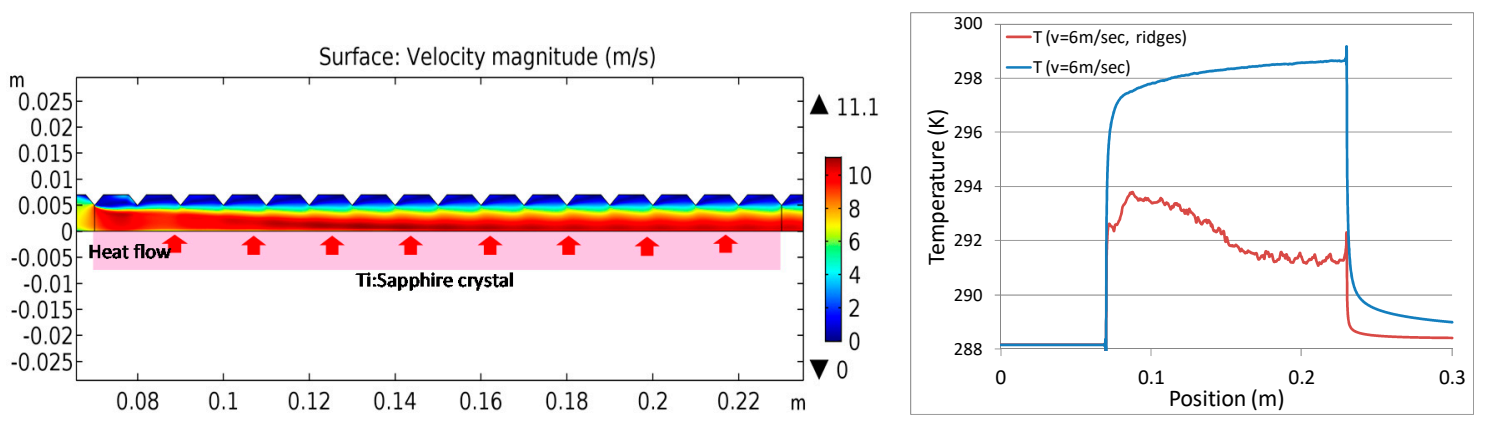

Figure 7. (Left) Fluid velocity distribution in the cooling channel, with triangular ridges on the upper wall. The triangular protrusions on the upper wall have a height of $2 \mathrm{~mm}$, base of $4 \mathrm{~mm}$, and distance of $10 \mathrm{~mm}$. The thickness of the channel between the ridges is $7 \mathrm{~mm}$. Other simulation conditions are the same as in Figure 6. (Right) Temperature profile along the channel position at the interface between the fluid and the Ti:Sapphire, for the smooth channel (blue line) and for the ridged channel (red line).

Such a configuration can be applied for the cooling of the disks in the reflection configuration, as the back face of the cooling channel is not crossed by the light beams, so it can be shaped to optimize heat exchange processes.

It can be noticed that the transversal flow configuration induces a temperature gradient in the cooling flow (and thus in the cooled surface of the Ti:Sa disk) in the flow direction as the fluid is heated along its path. This induces some amount of thermal aberration that is not radially symmetric, as would be desirable. On the other hand, the overall amount of this wavefront distortion is relatively small, at least for the highest flow speeds considered here, as it can be easily estimated. From the graph of Figure 7 it can be seen that the difference in the Ti:Sa surface temperature between the input edge and the output edge is about $1.6^{\circ} \mathrm{C}$. As the transverse heat flow in the Ti:Sa disk is negligible (due to the thin disk geometry), this temperature difference is directly added to the internal temperature distribution in the Ti:Sa. Accounting for the thermal expansion coefficient and for the value of $\mathrm{dn} / \mathrm{dT}$ of the Ti:Sa $\left(5 \times 10^{-5} / \mathrm{K}\right.$ and $1.5 \times 10^{-5} / \mathrm{K}$, respectively), the thermally induced optical path difference is about $0.4 \mu \mathrm{m}$ over a single pass (i.e., about $\lambda / 2$ ), with a roughly linear variation along the disk diameter. Moreover, this specific contribution to the wavefront aberration is stationary or at worst slowly varying in time, so it can be easily mitigated or canceled both by a proper geometrical arrangement (e.g., by having the flow propagating in alternate opposite directions in the disks) and by active wavefront correction optics, which are already considered in the design.

These investigations are to some extent preliminary, and a significantly broader work is required to obtain an executive design. Particularly relevant are the problems related to the vibrations induced by turbulences and by the pumping system, which could be detrimental for the beam pointing stability, in particular in the reflection geometry. Our major concern here was to assess the effectiveness of the water cooling approach at this unprecedented level of thermal load.

We notice here that according to the data reported in existing literature, gas cooling cannot provide a heat removal rate comparable with water cooling. Implementation of gas cooling would thus require unreasonably large heat exchange surfaces to obtain sufficient cooling, in particular at the envisaged P1 performance level. In more detail, currently the highest cooling performance obtained by this technique in the DiPOLE 100 system corresponds to the removal of about $4 \mathrm{~kW}$ of thermal load, with an available cooling surface of about $1450 \mathrm{~cm}^{2}$ (six Yb:YAG slabs with a cross-section of $11 \times 11 \mathrm{~cm}^{2}$, cooled on both sides) [15]; the average heat flow per unit cooling surface is less than $3 \mathrm{~W} / \mathrm{cm}^{2}$, and the film coefficient $k$ was estimated to about $0.17 \mathrm{~W}\left(\mathrm{~cm}^{2} \mathrm{~K}\right)$ [14]. On the other hand, the overall thermal load expected in the AMP3 module is about $10 \mathrm{~kW}$ (i.e., a factor 2.5-times larger than in DiPOLE 100), with an average heat flow per unit cooling surface as high as $25 \mathrm{~W} / \mathrm{cm}^{2}$ (i.e., an order of magnitude higher); the value of the heat transfer coefficient obtained with water cooling $\left(2.45 \mathrm{~W} /\left(\mathrm{cm}^{2} \mathrm{~K}\right)\right.$ is more than an order of magnitude larger than in the case of He cooling. We notice here that cryogenic $\mathrm{He}$ 
cooling was also recently employed for the cooling of a Ti:Sa multi-slab amplifier for the HAPLS system [16]; however, in that case the system runs at $3.3 \mathrm{~Hz}$, with a pump energy of about $60 \mathrm{~J}$, setting the average pump power to about $200 \mathrm{~W}$. This is much lower than the average pump power expected in the Eupraxia system for any of the amplification stages.

\section{Pump Laser Requirements and Available Technologies}

As outlined above, the laser amplification chains are composed of up to three individual amplifiers, labeled AMP1, AMP2, and AMP3 in the diagram in Figure 1. From the preliminary system design, the following pump energy requirements emerged, as summarized in Table 3.

Table 3. Pump energy requirements for the various amplification stages.

\begin{tabular}{ccc}
\hline Device & Pump Energy @ P0 (20 Hz) [J] & Pump Energy @ P1 (100 Hz) [J] \\
\hline AMP1 & 19.2 & 25.7 \\
AMP2 & 37.2 & 65.2 \\
AMP3 & 109 & 189 \\
\hline
\end{tabular}

Pump pulses must have a wavelength around 515-40 $\mathrm{nm}$ to match the absorption band of the Ti:Sapphire, and a pulse duration of several nanoseconds. Possible candidates as pump laser systems are the Premiumlite-YAG device developed by the French company Amplitude (formerly known as P60) and the DiPOLE 100 developed by the Central Laser Facility, Science and Technology Facility Council (STFC) Rutherford Appleton Laboratory in the United Kingdom.

The Premiumlite system is based on a chain of Nd:YAG disk amplifier heads (DAH) based on Nd:YAG, and currently operated with flash lamp pumping, liquid-cooled at room temperature. After conversion of the output to the second harmonic, the system delivers up to $60 \mathrm{~J}$ of pulse energy at $532 \mathrm{~nm}$, with a pulse duration of 5-6 ns. The system is modular, i.e., it can be scaled down by reducing the number of active heads. Currently the repetition rate is $10 \mathrm{~Hz}$, possibly limited by thermal management issues related to flashlamp pumping [21].

DiPOLE is a diode-pumped, solid state laser amplifier based on an end-pumped stack of ceramic Yb:YAG slabs, cooled by a flow of low-temperature, high-pressure helium gas. This technology was recently demonstrated at the $1 \mathrm{~kW}$ level with the DiPOLE100 system (STFC, UK; HiLASE, CZ), showing $100 \mathrm{~J}$ output energy at $10 \mathrm{~Hz}, 1030 \mathrm{~nm}$, with $>60 \mathrm{~J}$ expected conversion at $515 \mathrm{~nm}$ [1]. Current development plans are oriented toward a low energy, high repetition rate $(10 \mathrm{~J}$ at $100 \mathrm{~Hz})$ version of the system, whereas the frequency upscale of the $100 \mathrm{~J}$ version is probably challenged by thermal management issues.

\section{Pulse Compression and Beam Transport System}

The pulse compressor and the beam transport system also pose specific problems because of the high average power level handled by the pulse compressor and the high pointing stability required for the interaction with the plasma target.

A general scheme for this part of the apparatus is depicted in Figure 8. The design of the whole subsystem has to be compliant with the final goal of recompressing the $125 \mathrm{~J}$ CPA pulse coming from the last amplification stage down to 20-60 fs on-target (depending on the laser system, see Table 1 for details). 


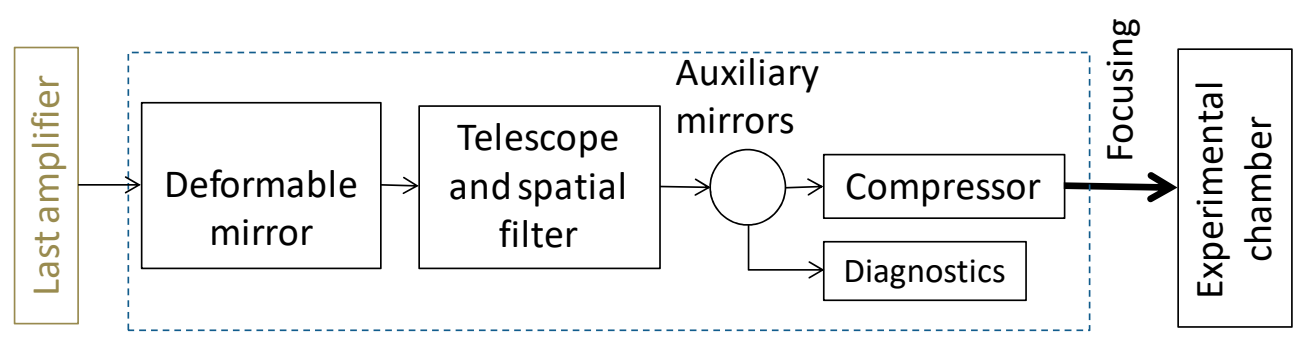

Figure 8. General transport and compression scheme.

The main system elements are:

(a) a deformable mirror for the optimization of the phase front of the beam in a specific plane in the compressor, to keep the phase front compatible with the required compression;

(b) a beam expander with a given magnification, to relay the image plan on the deformable mirror, which also includes a spatial filter implemented by placing a pinhole at the focal spot;

(c) a temporal compression stage.

The spatial filter is required to remove high spatial frequencies from the beam (due for instance to inhomogeneities in the Ti:Sa crystal), which would otherwise induce hot spots in the beam profile along the transport chain. In any case, the diameter of the pinhole in the spatial filter will be much larger than the spot size (i.e., by a factor between 2 and 10); moreover, the spatial filter is placed downstream with respect to the deformable mirror, which manages both the wave front correction and the pointing stabilization, so that overall there is no risk that the pinhole will be damaged by the laser beam.

Between the spatial filter and the compressor, a possible series of 2 mirrors for polarization rotation or simple beam transport towards the compressor entrance can be considered, also to allow the detection of beam leakage for diagnostics purpose. This stage actually depends on the choice of compressor gratings, and it will be needed for gold gratings, as explained below.

The next section will describe for each point the available technologies and schemes, and the choice of the best option.

\subsection{Compressors}

At each compressor, the average input power and output pulse parameters will be:

Laser 1: 1.2 kW (12 J @ $100 \mathrm{~Hz})$, needed 20 fs after compression (62 nm bandwidth), spectral acceptance required: $140 \mathrm{~nm}$

Laser 2: 4 kW (40 J @ $100 \mathrm{~Hz}$ ), needed 20 fs after compression (62 nm bandwidth), spectral acceptance required: $140 \mathrm{~nm}$

Laser $313 \mathrm{~kW}$ (130 J @ $100 \mathrm{~Hz}$ ), needed $60 \mathrm{fs}$ after compression ( $23 \mathrm{~nm}$ bandwidth), spectral acceptance required: $60 \mathrm{~nm}$

Laser induced damage threshold (LIDT) of gratings limits the laser fluence arriving on the compressor for each laser system to $100 \mathrm{~mJ} / \mathrm{cm}^{2}$, and thus the average intensity impinging on the compressors to $10 \mathrm{~W} / \mathrm{cm}^{2}$. While gratings in air might handle this intensity, it is not at all obvious whether they can do it in a vacuum. The only study available so far on gold grating cooling in air was carried out [22] at LLNL. For these reasons, given the current lack of studies on this issue, the baseline choice for the EuPRAXIA compressor grating material is gold gratings without resin, which requires the study of a cooling strategy. The absence of resin between the gold layer and the substrate might induce less deformation in the gratings, but there is still no established evidence on this and an accurate study is therefore mandatory. Indeed, some new grating technology could make cooling unnecessary, thus simplifying significantly the complexity of the system.

Experimental tests were carried out on heating effects of the gratings of an ultrashort pulse compressor using a $\mathrm{kW}$ laser diode as the thermal source. The compressor is installed in the front end 
hall of the Apollon laser $[23,24]$ and allows the compression of $\mathrm{mJ}$ level pulses at $<17 \mathrm{fs}$ in air. Based on wavelength-dependent wavefront measurements, the spatiotemporal impact on the compressed pulses was evaluated.

The experimental setup is shown in Figure 9. A typical folded gold grating based compressor is used. In order to experimentally reproduce the high average power conditions on the compressor gratings, a $1.2 \mathrm{~kW}$ laser diode at $980 \mathrm{~nm}$ was used as the thermal source. The heating beam from the diode is first split into twin beams via a polarizer beam splitter (PBS) and each one is directed precisely on the input and output sections of the first compressor grating (G1).

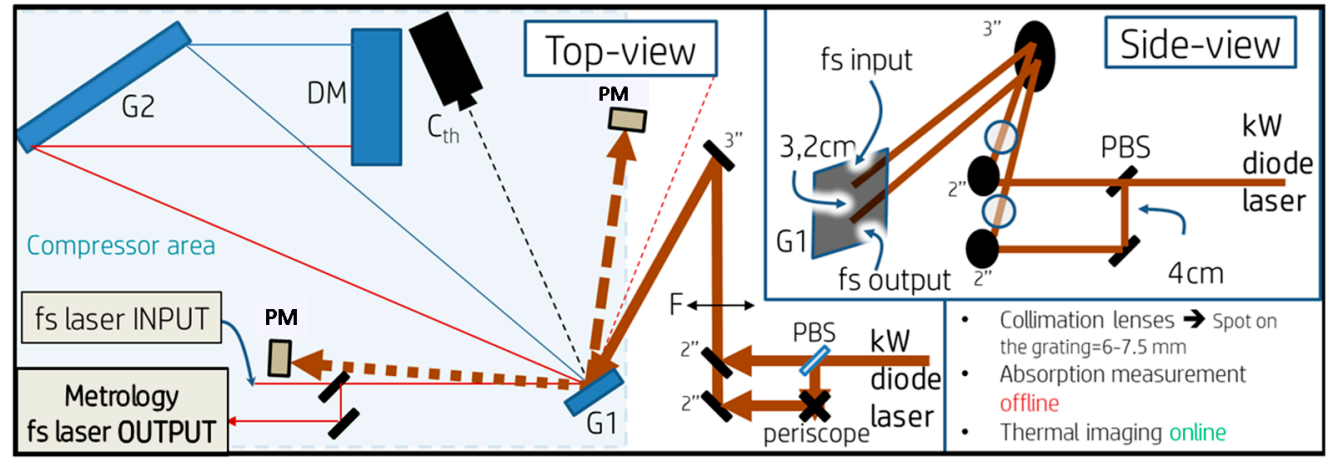

Figure 9. Top-view and side-view of the experimental set-up. Compressor Area: $\mathrm{PM}=$ power meter; $\mathrm{C}_{\mathrm{th}}=$ Thermal camera; G1 = first compressor grating; G2 = second compressor grating (gold, 56, 14801/mm); DM = Dihedral Mirror. Diode Area: F = imaging lens; PBS = Polarizing Beam Splitter.

An imaging system allows a perfect overlap of the diode heating beam on the ultrashort beam (Figure 10a). A thermal camera is used in parallel to measure the local spatial distribution of the temperature increase (Figure 10b,c). The metrology used includes a wavefront sensor (Phasics SID4), achromatic near and far field sensors, as well as a single shot autocorrelator and a Wizzler for the estimation of the impact on the pulse duration.

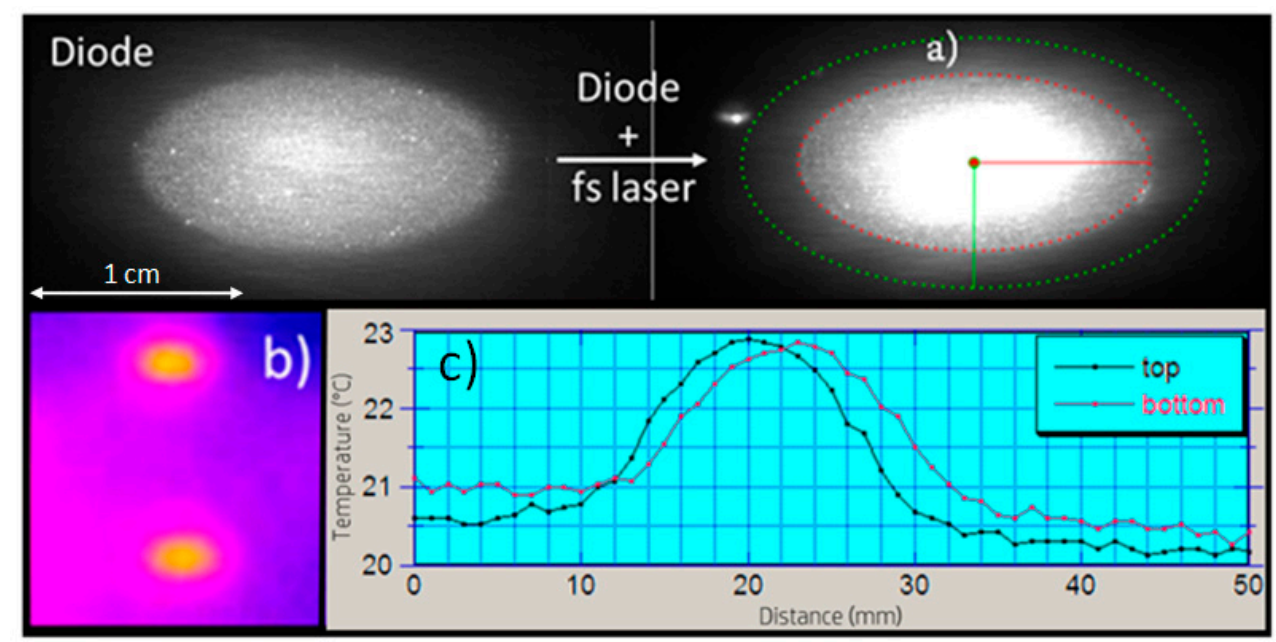

Figure 10. (a) Image of the heating diode laser beam and of the fs laser beam on G1. The laser diode beam has a top hat profile, with a spot size of $0.82 \mathrm{~cm} \times 1.63 \mathrm{~cm}$ (surface $\sim 1 \mathrm{~cm}^{2}$ ). The edge of the diode beam is evidenced by a dashed red line, and the edge of the fs beam by a dashed green line. (b) G1 thermal image for 130W sent on each spot (see above). (c) Temperature profiles for the beam spots (b); left profile: input spot; right profile: output spot.

For different values of the average power $(0-130 \mathrm{~W})$ sent on $\mathrm{G} 1$, the wavefront (peak-to-valley and RMS values in $\lambda$ units), Strehl ratio, defocus, and all the higher order induced aberrations 
were measured. A significant degradation of the spatial profile of the beam was measured, and an accentuated astigmatism was noted, induced by the gratings when they are heated. The focal spot is asymmetrically distorted, and the first measurements suggest a non-negligible difference in this degradation for different wavelengths. (Figure 11c).

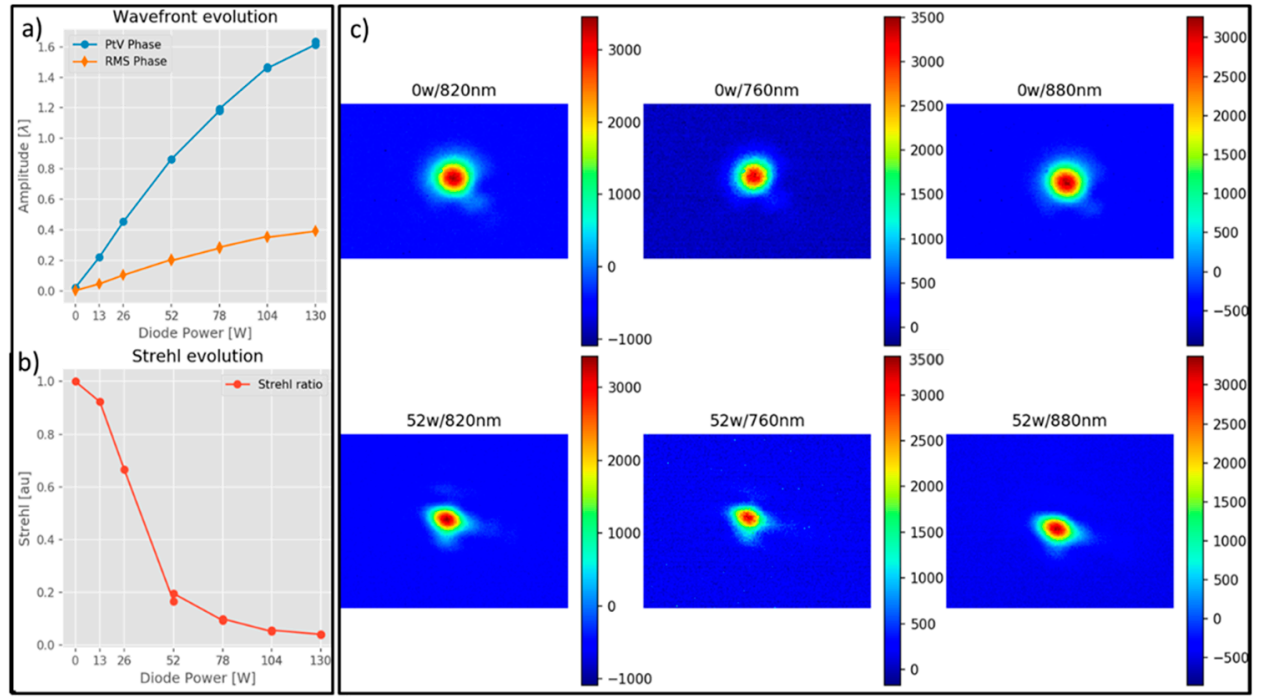

Figure 11. The (increasing) power sent on both input and output spots. (a) Wavefront degradation. (b) Strehl ratio degradation-the graph reports the ratio between the Strehl ratio at a given power and that at zero heating power. (c) Upper line: far field at $760 \mathrm{~nm}, 820 \mathrm{~nm}, 880 \mathrm{~nm}$ for $0 \mathrm{~W}$ heating power. Lower line: far field at $760 \mathrm{~nm}, 820 \mathrm{~nm}, 880 \mathrm{~nm}$ for $52 \mathrm{~W}$ heating power. Pairs of spots at each wavelength are normalized at the same peak intensity to allow a clearer comparison of the spot shapes. Spots at different wavelengths cannot be compared directly due to the different shape of the unperturbed beam.

\subsection{Beam at the Interaction Point}

In previous work [6] an overview was given of the transport and compression strategy from the compressor output to the interaction point.

A recent work was dedicated to address viable solutions for the interaction point spatial beam diagnostics and the pointing stability diagnostics, which is the final part of the chain until the interaction point (as identified in the scheme in Figure 8). The block diagram in Figure 12 shows the requirements needed to fully characterize and try to correct the beam characteristics in the interaction point.

\section{Two purposes}

\section{characterization of:}

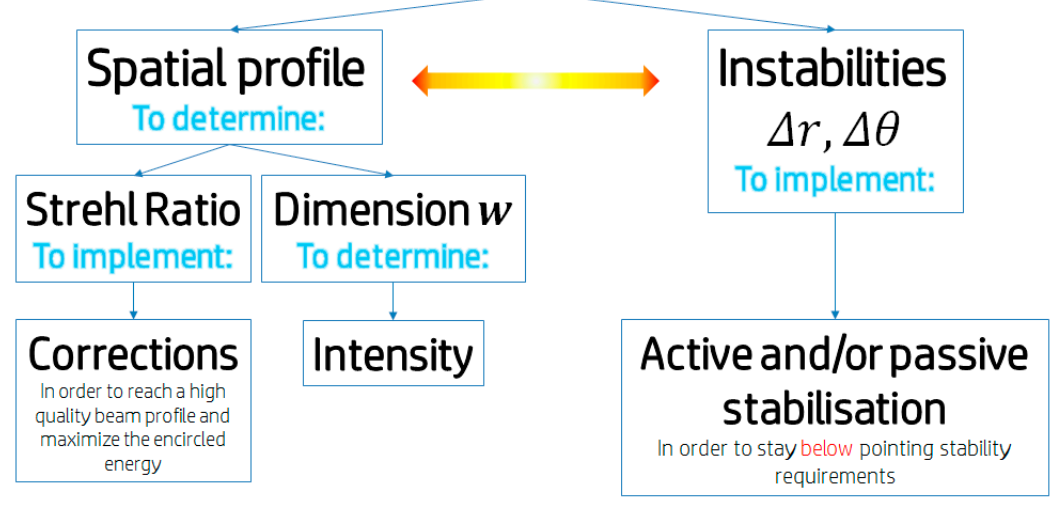

Figure 12. Preliminary flow chart of the laser stability diagnostics and control in the focal point. 
Spatial beam diagnostics in the focal plane are necessary in order to measure and improve the beam spatial quality, in a closed loop with the deformable mirror, and in order to estimate the beam intensity achieved during the acceleration experiment.

Focusing conditions on the target require a beam pointing stability of the order of hundreds of nano-radians. This value was set up considering that the pointing instability of the output accelerated electron beam will be influenced by the laser pointing instability. Indeed, focusing of Laser 3 down to a spot of around $100 \mu \mathrm{m}$ starting from a near field beam diameter of tens of $\mathrm{cm}$ requires focal lengths of tens of meters. Moreover, in the case of the $5 \mathrm{GeV}$ acceleration laser, capillaries might be used, and it is important not to damage them during the acceleration phase with unstable pointing on the focal plane. Very long focal lengths cause transverse shifts (hundreds of microns) on the focal plane, even if the laser angular stability is below the micro radiant level (which is normally considered very stable). Several solutions are being investigated to solve this issue, which is still open in all the facilities worldwide. A diagnostic tool is needed in order to measure angular stabilities below the micro radians level, and even more importantly, a diagnostic tool that is able to measure angular fluctuations above $100 \mathrm{~Hz}$ frequency. Some facilities have dedicated optical systems for this purpose [25], but only in certain positions along the laser chain. A simple and easy to use diagnostic tool is needed in order to evaluate the entire line of a high-peak, high-average power laser system.

The most used method to measure spatial quality on the far field and pointing stability up to a repetition rate of tens of $\mathrm{Hz}$ is to image the focal plane where the laser beam is focused.

In the framework of the Eupraxia activities, a device for the measurement of beam pointing stability and beam shape has been tested (Figure 13).

This module is so compact that it can be placed on the beam path to make a magnified image of the laser spot in the focal plane and around the focal region, to study the laser beam spatial quality and stay in the chamber during laser shots. Its shielding has been studied to survive radiations and EMP during the shots, and special care has been taken in the choice of materials to be complaint with our residual gas analysis criteria so that it will not contaminate the final optics coating. The device can, therefore, stay in the interaction chamber during shots and can be used to make on-shot measurements. The laser system can operate at full energy (thus being subjected to realistic thermal load effects), but the beam after the amplification stage must be attenuated because only a few tens of microjoules can enter the camera module.

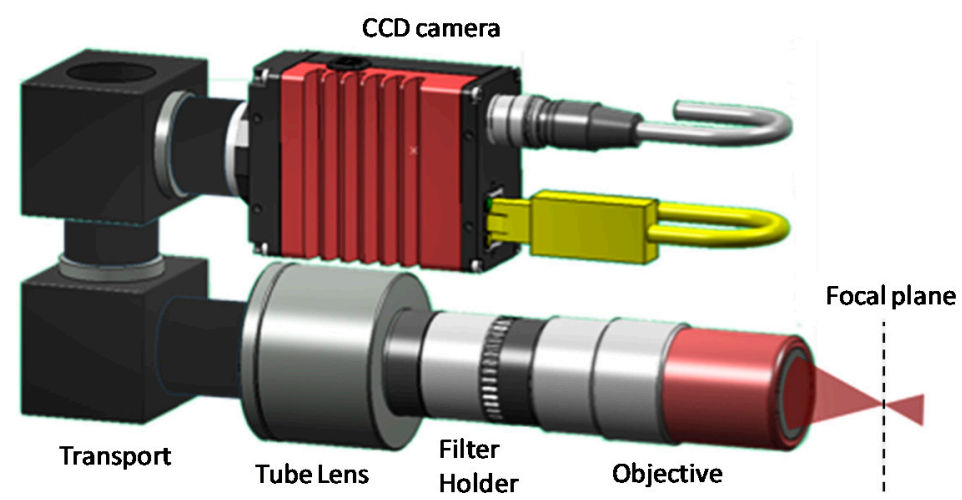

Figure 13. Design and experimental prototype of the module for focal spot quality and stability measurements.

In Apollon, a reflection-type attenuator has been studied to reduce the beam up to 6 orders of magnitude, plus an absorption-type attenuator to be placed just after. Filters are made of Schott VG9 colored glass, which has almost spectrally flat transmission around $800 \mathrm{~nm}$, as seen from the data in Figure 14. Tests have shown that the wavefront distortion induced by the filters is less than $\lambda / 10$. 

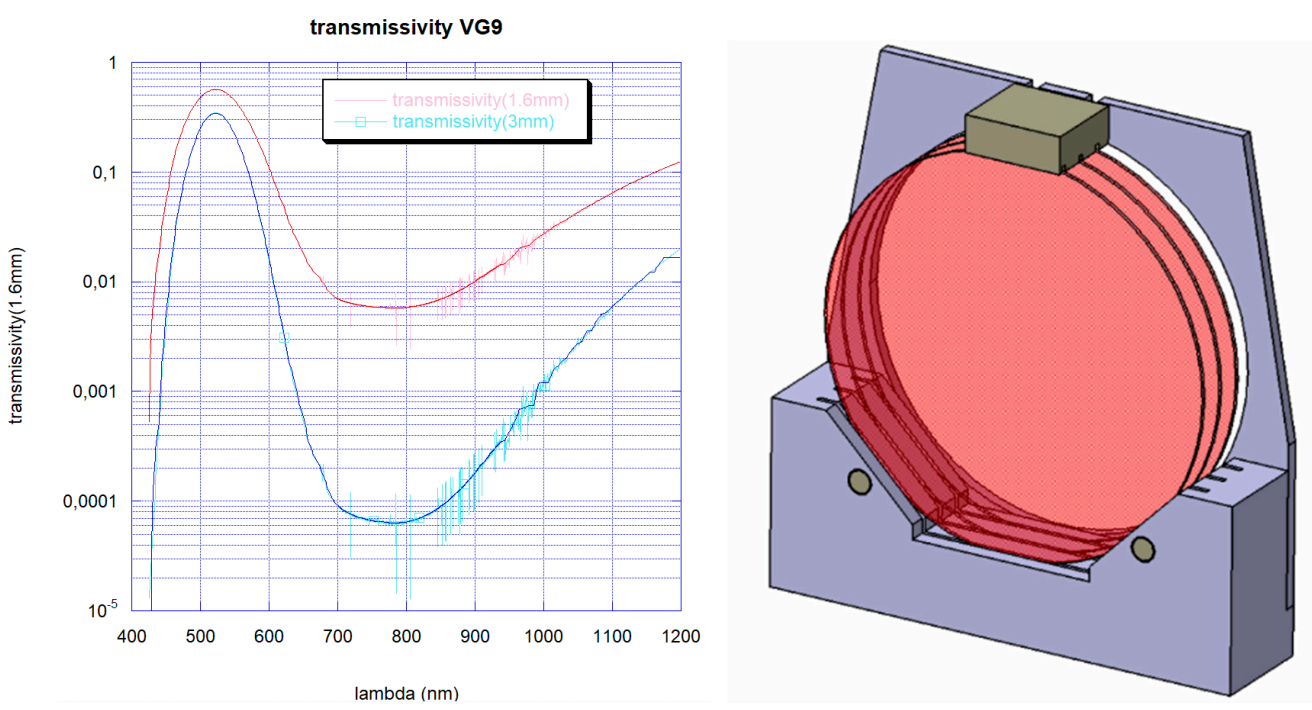

Figure 14. (Left) VG9 transmission spectra, measured with a 3-mm thick sample (blue curve) and a 1.6-mm thick sample (red curve). (Right) Mounting for the VG9 filters. Filter diameter is $160 \mathrm{~mm}$, thickness $1.6 \mathrm{~mm}$.

\section{Conclusions}

The requirements for the EuPRAXIA laser driver make its design and its realization a very challenging task. These requirements set unprecedented laser performance, never achieved before by any existing laser system worldwide. Nonetheless, recent advancements in laser technology are making the achievement of these performances feasible. On the basis of the requirements, and considering both the available technologies and those at high TRL and reasonable availability in the next few years at an industrial level, a baseline for the system architecture and for the key technologies was devised. The system concept delivered so far combines most, if not all, of the highly advanced and proven technologies and aims to deliver performances well beyond those of systems that already exist, or those currently under construction. Some critical aspects (for instance those related to the amplifiers cooling, the thermal deformation of the gratings, and the pointing stability) have been discussed and are being addressed both by means of simulations and by means of dedicated experimental studies. With this conceptual design, EuPRAXIA will provide a credible step towards reliable operation of a laser-driven plasma accelerator, fostering the development of the European laser industry and launching a disruptive advancement in high power, laser-based sciences and applications.

Author Contributions: Conceptualization, L.G., F.M., G.T., L.L., Z.M.; Investigation, G.T., Z.M., L.L., F.M., M.V., B.P. and L.G.; Supervision, F.M. and L.G.; Writing-original draft preparation, G.T., Z.M. and L.L.; Writing-review \& editing, G.T., M.V., B.P., L.L. and L.G. Funding acquisition, F.M. and L.G.

Funding: The research leading to these results has received funding from the European Union Horizon 2020 Research and Innovation Program under Grant Agreement No. 653782 EuPRAXIA. This project has received funding from the CNR funded Italian research Network ELI-Italy (D.M. n. 631 08.08.2016).

Acknowledgments: We gratefully acknowledge P. Mason and K. Ertel of the Central Laser Facility, A. Bayramian, C. Siders, and C. Haefner of the Lawrence Livermore National Laboratory, and F. Falcoz of Amplitude Technologies for fruitful discussion on the laser developments.

Conflicts of Interest: The authors declare no conflict of interest.

\section{References}

1. Mason, P.; Divoký, M.; Ertel, K.; Pilař, J.; Butcher, T.; Hanuš, M.; Banerjee, S.; Phillips, J.; Smith, J.; Vido, M.D.; et al. Kilowatt average power 100j-level diode pumped solid state laser. Optica 2017, 4, 438-439. [CrossRef] 
2. Sistrunk, E.F.; Spinka, T.; Bayramian, A.; Armstrong, O.; Baxamusa, S.; Betts, S.; Bopp, D.; Buck, D.; Charron, K.; Cupal, J.; et al. All diode-pumped, high repetition-rate advanced petawatt laser system (HAPLS). In Proceedings of the CLEO: Science and Innovations 2017, San Jose, CA, USA, 14-19 May 2017.

3. Danson, C.; Hillier, D.; Hopps, N.; Neely, D. Petawatt class lasers worldwide. High Power Laser Sci. Eng. 2015, 3, e3. [CrossRef]

4. Nakamura, K.; Mao, H.S.; Gonsalves, A.J.; Vincenti, H.; Mittelberger, D.E.; Daniels, J.; Magana, A.; Toth, C.; Leemans, W.P. Diagnostics, control and performance parameters for the bella high repetition rate petawatt class laser. IEEE J. Quantum Electron. 2017, 53, 1-21. [CrossRef]

5. Walker, P.A.; Alesini, P.D.; Alexandrova, A.S.; Anania, M.P.; Andreev, N.E. Horizon 2020 EuPRAXIA design study. J. Phys. Conf. Ser. 2017, 874, 1-8. [CrossRef]

6. Gizzi, L.A.; Koester, P.; Labate, L.; Mathieu, F.; Mazzotta, Z.; Toci, G.; Vannini, M. A viable laser driver for a user plasma accelerator. Nucl. Instrum. Methods Phys. Res. A 2018, 58-66. [CrossRef]

7. Tomassini, P.; De Nicola, S.; Labate, L.; Londrillo, P.; Fedele, R.; Terzani, D.; Gizzi, L.A. The resonant multi-pulse ionization injection. Phys. Plasmas 2017, 24, 103120. [CrossRef]

8. Siders, C.W.; Siders, J.L.W.; Taylor, A.J.; Park, S.J.; Weiner, A.W. Efficient high-energy pulse-train generation using a 2n-pulse Michelson interferometer. Appl. Opt. 1998, 37, 5302-5305. [CrossRef]

9. Dromey, B.; Zepf, M.; Landreman, M.; O'Keeffe, K.; Robinson, T.; Hooker, S.M. Generation of a train of ultrashort pulses from a compact birefringent crystal array. Appl. Opt. 2007, 46, 5142-5146. [CrossRef]

10. Cowley, J.; Thornton, C.; Arran, C.; Shalloo, R.J.; Corner, L.; Cheung, L.; Gregory, C.D.; Mangles, S.P.D.; Matlis, N.H.; Symes, D.R.; et al. Excitation and Control of Plasma Wakefields by Multiple Laser Pulses. hao 2017, 119, 044802. [CrossRef]

11. Vantaggiato, G.; Labate, L.; Tomassini, P.; Gizzi, L.A. Modelling of pulse train generation for resonant laser wakefield acceleration using a delay mask. Nucl. Instrum. Meth. Phys. Res. A 2018, 909, 114-117. [CrossRef]

12. Morice, O. Miró: Complete modeling and software for pulse amplification and propagation in high-power laser systems. Opt. Eng. 2003, 42, 1530-1542. [CrossRef]

13. Nagymihaly, R.S.; Cao, H.; Papp, D.; Hajas, G.; Kalashnikov, M.; Osvay, K.; Chvykov, V. Liquid-cooled Ti: Sapphire thin disk amplifiers for high average power 100-TW systems. Opt. Express 2017, 25, 6664-6677. [CrossRef] [PubMed]

14. Mason, P.D.; Fitton, M.; Lintern, A.; Banerjee, S.; Ertel, K.; Davenne, T.; Hill, J.; Blake, S.P.; Phillips, P.J.; Butcher, T.J.; et al. Scalable design for a high energy cryogenic gas cooled diode pumped laser amplifier. Appl. Opt. 2015, 54, 4227-4238. [CrossRef]

15. Banerjee, S.; Mason, P.D.; Ertel, K.; Phillips, P.J.; De Vido, M.; Chekhlov, O.; Divoky, M.; Pilar, J.; Smith, J.; Butcher, T.; et al. $100 \mathrm{~J}-$ level nanosecond pulsed diode pumped solid state laser. Opt. Lett. 2016, 41, 2089-2092. [CrossRef] [PubMed]

16. Haefner, C.L.; Bayramian, A.; Betts, S.; Bopp, R.; Buck, S.; Cupal, J.; Jarboe, J. High average power, diode pumped petawatt laser systems: A new generation of lasers enabling precision science and commercial applications. In Research Using Extreme Light: Entering New Frontiers with Petawatt-Class Lasers III; International Society for Optics and Photonics: Washington, WA, USA, 2017.

17. Chvykov, V.; Nagymihaly, R.S.; Cao, H.; Kalashnikov, M.; Osvay, K. Design of a thin disk amplifier with extraction during pumping for high peak and average power Ti: Sa systems (EDP-TD). Opt. Express 2016, 24, 3721-3733. [CrossRef] [PubMed]

18. Chu, Y.; Liang, X.; Yu, L.; Xu, Y.; Xu, L.; Ma, L.; Lu, X.; Liu, Y.; Leng, Y.; Li, R.; et al. High-contrast 2.0 Petawatt Ti: Sapphire laser system. Opt. Express 2013, 21, 29231-29239. [CrossRef] [PubMed]

19. Gizzi, L.A.; Giove, D.; Altana, C.; Brandi, F.; Cirrone, P.; Cristoforetti, G.; Fazzi, A.; Ferrara, P.; Fulgentini, L.; Koester, P.; et al. New Line for Laser-Driven Light Ions Acceleration and Related TNSA Studies. Appl. Sci. 2017, 7, 984. [CrossRef]

20. Ferrara, P.; Ciofini, M.; Esposito, L.; Hostaša, J.; Labate, L.; Lapucci, A.; Pirri, A.; Toci, G.; Vannini, M.; Gizzi, L.A. 3-D numerical simulation of Yb: YAG active slabs with longitudinal doping gradient for thermal load effects assessment. Opt. Express 2014, 22, 5375-5386. [CrossRef]

21. Charalambidis, D.; Chikán, V.; Cormier, E.; Dombi, P.; Fülöp, J.A.; Janáky, C.; Lepine, F. The extreme light infrastructure-Attosecond light pulse source (ELI-ALPS) project. In Progress in Ultrafast Intense Laser Science XIII; Springer: Basel, Switzerland, 2018; pp. 181-218. 
22. Alessi, D.A.; Sistrunk, E.; Nguyen, H.T.; Rosso, P.A.; Spinka, T.; Aasen, M.D.; Herriot, S.; Britten, J.A.; Haefner, C. A Compressor for High Average Power Ultrafast Laser Pulses with High Energies. In Proceedings of the Conference on Lasers and Electro-Optics, San Jose, CA, USA, 14-19 May 2017.

23. Papadopoulos, D.; Zou, J.; Le Blanc, C.; Chériaux, G.; Georges, P.; Druon, F.; Audebert, P. The Apollon 10 PW laser: Experimental and theoretical investigation of the temporal characteristics. High Power Laser Sci. Eng. 2016, 4, E34. [CrossRef]

24. Papadopoulos, D.N.; Ramirez, P.; Genevrier, K.; Ranc, L.; Lebas, N.; Pellegrina, A.; Le Blanc, C.; Monot, P.; Martin, L.; Zou, J.P.; et al. High-contrast 10 fs OPCPA-based front end for multi-PW laser chains. Opt. Lett. 2017, 42, 3530-3533. [CrossRef]

25. Canuel, B.; Genin, E.; Mantovani, M.; Marque, J.; Ruggi, P.; Tacca, M. Sub-nanoradiant beam pointing monitoring and stabilization system for controlling input beam jitter in gravitational wave interferometers. Appl. Opt. 2014, 53, 2906-2916. [CrossRef] [PubMed]

(C) 2019 by the authors. Licensee MDPI, Basel, Switzerland. This article is an open access article distributed under the terms and conditions of the Creative Commons Attribution (CC BY) license (http://creativecommons.org/licenses/by/4.0/). 